\title{
Acute Infection of Chinese Macaques by a CCR5-Tropic SHIV Carrying a Primary HIV-1 Subtype B' Envelope
}

\author{
Haibo Wang, PhD, *† Ke Zhuang, PhD, * Li Liu, PhD, † Zhijiao Tang, MD, $\neq$ Juanjie Tang, PhD, * \\ Po Tien, PhD, * Linqi Zhang, PhD, $\S^{\|}$and Zhiwei Chen, PhD, DVM†
}

\begin{abstract}
The increasing prevalence of HIV-1 subtype B in China and Southeast Asia calls for efforts to develop a relevant animal model to study viral transmission and pathogenesis. Because there are significant differences between subtype B HIV-1 and other chimeric simian/human immunodeficiency viru (SHIVs) in the env gene, a novel SHIV, designated SHIVB' ${ }_{\text {wHU, }}$, was generated by replacing counterparts of SHIVSF33 with tat/rev/vpu/env genes derived from a primary, CCR5-tropic, subtype B' HIV-1 strain of a Chinese patient. SHIVB' ${ }_{\text {wHU }}$ was able to replicate in rhesus peripheral blood mononuclear cells and used CCR5 as its major coreceptor. Moreover, after serial passages in Chinese macaques, the in vivo infectivity of SHIVB' ${ }_{\text {wHU }}$ was enhanced, yet no significant sequence changes were found in viral envelopes, and the virus did not change its CCR5tropism. $\mathrm{CD}^{+}$T-cell loss, however, was found in the intraepithelial lymphocytes of small intestines of infected macaques. Our findings have implications in understanding the early pathogenesis of SHIVB' ${ }_{\text {wHU }}$ in Chinese macaques.
\end{abstract}

Key Words: acute infection, animal model, Chinese macaques, SHIV, subtype B'

(J Acquir Immune Defic Syndr 2009;00:000-000)

Received for publication August 5, 2009; accepted November 18, 2009. From the *AIDS Center and Modern Virology Research Center, State Key Laboratory of Virology, College of Life Sciences, Wuhan University, People's Republic of China; †AIDS Institute, Li Ka Shing Faculty of Medicine, The University of Hong Kong, Hong Kong SAR, People's Republic of China; $\$$ Laboratory Animal Center, State Key Laboratory of Virology, Wuhan University, People's Republic of China; §AIDS Research Center, Institute of Pathogen Biology, Chinese Academy of Medical Sciences, Beijing, People's Republic of China; and "Comprehensive AIDS Research Center, Tsinghua University, Beijing, People's Republic of China.

Supported by the National Basic Research Program of China (the 973 project 2006CB504208), the National 11th 5-year project 2008ZX10O01-011, Hong Kong research grant council (HK-RGC762208 and HKRGC762209 to ZC), and the University Development Fund of the University of Hong Kong to its AIDS Institute.

The authors declare no financial or commercial conflict of interest.

The first 2 authors H.W. and K.Z. contributed equally to this work.

Supplemental digital content is available for this article. Direct URL citations appear in the printed text and are provided in the HTML and PDF versions this article on the journal's Web site (www.jaids.com).

Correspondence to: Zhiwei Chen, PhD, AIDS Institute, Li Ka Shing Faculty of Medicine, The University of Hong Kong, 21 Sassoon Road, Pokfulam, Hong Kong S.A.R., People's Republic of China (e-mail: zchenai@hku.hk). Copyright (c) 2010 by Lippincott Williams \& Wilkins

\section{INTRODUCTION}

Human immunodeficiency virus type one (HIV-1) epidemic in China is largely driven by several high-risk factors. ${ }^{1}$ One of the risk factors is paid blood donation (PBD), which was initially identified in Henan province, where many poor farmers were infected through illegal blood collection practices without adequate screening and sterilization procedures. ${ }^{2,3}$ Many of the infections occurred when contaminated blood cells were transfused back to donors once the plasma had been removed from the pooled blood. Over the past years, such activities, which peaked between 1992 and 1996, have caused hundreds of thousands of new infections among paid blood donors in rural areas in Henan and neighboring provinces including Anhui, Hubei, Hebei, Shaanxi, and Shanxi, ${ }^{2,4}$ creating the tragic "AIDS villages" 5,6 and attracting tremendous public attention. ${ }^{7,8}$ Although illegal PBD has been banned by the Chinese government, the increasing prevalence of HIV-1 subtype B' through other risk behaviors including unprotected sexual contacts still calls for efforts to fight the epidemic.

To fulfill the goal of controlling HIV-1 dissemination from high-risk groups into general populations, ${ }^{9,10}$ there is a need to understand the viral transmission and to develop an effective preventive means. Because many preventive means cannot be tested in humans, it is desirable to develop a relevant animal model. Therefore, a lot of efforts have been put on the development of the chimeric simian/human immunodeficiency virus (SHIV). ${ }^{11-13}$

Most of currently available SHIVs utilize envelope genes derived from HIV-1 subtype B strains, either from laboratory-adapted, syncytium-inducing, T-tropic viruses $\left(\mathrm{HIV}-1_{\mathrm{HXB} 2}\right.$ and $\left.\mathrm{HIV}-1_{\mathrm{NL} 43}\right)$ or from primary, nonsyncytiuminducing, M-tropic (HIV-1 $\left.{ }_{\mathrm{SF} 162}\right)$, syncytium-inducing T-tropic (HIV-1 $\left.1_{\mathrm{SF} 33}\right)$ and dual-tropic (HIV-1 $1_{89.6}$ and HIV$\left.1_{\mathrm{DH} 12}\right)$ isolates. ${ }^{14,15}$ In addition, a few nonsubtype B SHIVs were generated for subtype $\mathrm{C}$ and $\mathrm{E}$ including the SHIV $_{\text {CHN19 }}$ that we previously generated to study the major HIV-1 epidemic caused by subtype C and CB recombinant viruses in China. ${ }^{16-18}$ Till now, however, no SHIV has been generated based on the envelope of subtype B' HIV-1, another major epidemic strain in China and Southeast Asia. Moreover, it remains unknown whether or not a SHIV generated from a freshly isolated human HIV-1 would be pathogenic in vivo during acute phase of infection. Here, we report the construction and characterization of a new SHIV $_{B^{\prime} \text { wHU }}$ both in vitro and in vivo. 


\section{MATERIAL AND METHODS}

\section{Amplification of HIV-1 tat/rev/vpu/env Genes}

The genomic DNA was extracted from peripheral blood mononuclear cells (PBMCs) of an HIV-1-infected PBD $\left(02 \mathrm{HN}_{\mathrm{SMX} 2}\right){ }^{2}$ The HIV-1 tat/rev/vpu/env fragment was amplified from genomic DNA by a nested polymerase chain reaction (PCR) using the EXPAND high-fidelity DNA polymerase according to the manufacturer's specifications (BoehringerMannheim, IN). The outer primer pair is $4759 \mathrm{~F} 1\left(5^{\prime}-\mathrm{GTT}\right.$ TTT CAG AAT CTG CTA TAA GAA ATG CC-3'), BU5 (B) (5' -CTC TAG TTA CCA GAG TCA CAC AAC AGA CGG GCA CAC AC-3') and the inner primer pair is $\mathrm{VprA}$ (5'-TGCC GAATTCGCATGC TA TAG A TAG AGG AGA GCA AGA AAT GGA G -3') and EnvB (5' -TGCC CTCGAG CT TAT AGT AAA GCC CTT TCG AGG-3'), where the introduced stop codons are underlined and the restriction enzyme sites (EcoRI, SphI, and Xhol) are italicized. Products from the second-round amplifications were purified from agarose gels using QIAquick gel extraction kit (Qiagen, Valencia, CA).

\section{Construction of SHIV $_{B^{\prime} W H U}$}

The env sequence was subjected to the phylogenetic analysis as previously described. ${ }^{16}$ PVP-1 plasmid contains the $5^{\prime}$-half $\mathrm{SIV}_{\mathrm{mac} 239}$ genome from the $5^{\prime}$ long terminal repeat, a generous gift of Dr Paul Luciw. 3' half $\mathrm{vpu}^{+}-\mathrm{B}^{\prime}{ }_{\text {wHu }}$ plasmid $\left(3^{\prime}-\mathrm{p} 02 \mathrm{HN}_{\mathrm{SMX} 2}-\mathrm{B}^{\prime}{ }_{\mathrm{WHU}}\right)$ contains the $3^{\prime}$-half of $\mathrm{SHIV}_{\mathrm{B}}$ 'wHU genome. The $3^{\prime}-\mathrm{p} 02 \mathrm{HN}_{\mathrm{SMX} 2}-\mathrm{B}^{\prime}{ }_{\text {wHU }}$ plasmid was made by replacing counterparts of $\mathrm{SHIV}_{\mathrm{SF} 33}$ (a generous gift of Dr Cecilia Cheng-Mayer) with newly obtained subtype B' tat/rev/vpu/env genes. To make a replication-competent recombinant SHIV ${ }_{\mathrm{B}}$ 'WHU, linear 5'-PVP-1 and $3^{\prime}-\mathrm{p} 02 \mathrm{HN}_{\mathrm{SMX2}}{ }^{-}$ $\mathrm{B}^{\prime}{ }_{\text {WHU }}$ plasmids were co-transfected into $293 \mathrm{~T}$ cells using Effectene Transfection Reagent (Qiagen, Valencia, CA). Forty-eight hours later, $1 \times 10^{7} \mathrm{CEM} \times 174-5.25 \mathrm{M} 7$ cells in DMEM media were placed into the $60 \mathrm{~mm}$ dishes containing the transfected $293 \mathrm{~T}$ cells and supernatant. The culture supernatants were harvested for p27 measurement and used to infect macaque PBMCs. Some transfected 293T cells were also subjected to the cell fusion assay to test the expression and phenotype of Env as we previously described. ${ }^{16}$

\section{Genetic Analysis of Amplified HIV-1 Genes}

Full-length tat/rev/vpu/env amplicon was cloned into the expression vector pcDNA-I/Amp (Invitrogen, Carlsbad, CA) and was subsequently sequenced. The full env sequence was subjected to phylogenetic analysis. ${ }^{1,2}$ The phylogenetic tree was generated using the neighbor-joining method implemented in the ClustalX1.8 program. The tree was plotted using the TreeView program. ${ }^{19}$ The SimPlot and BootScan analysis was used to determine the possible intersubtype recombination. ${ }^{20}$

\section{Replication of SHIV $\mathrm{B}^{\prime} W H \mathrm{in}$ in Chinese Macaque PBMCs}

Macaque blood was obtained from healthy adult Chinese rhesus monkeys that were provided by Wuhan University Laboratory Animal Center. PBMCs were separated from the whole blood by centrifugation through Ficoll-Paque PLUS (GE Healthcare, Piscataway, NJ) density gradients. To test the infectivity of newly generated SHIV $_{\mathrm{B}^{\prime} \text { wHU }}$ strain, $1 \times 10^{7}$ stimulated macaque PBMCs were infected by cell-free virus with an equal inoculum (p27 titer: $0.5 \mathrm{ng} / \mathrm{mL}$ ). The infected PBMCs were cultured in 10\% FBS RPIM-1640 containing $20 \mathrm{U}$ IL-2. On day 5, 10 and 15 postinfection, the viral supernatants were harvested for p27 measurement. The p27 level was measured using a kit purchased from ZeptoMetrx Corporation (Buffalo, New York, NY).

\section{FACS ANALYSIS}

The number of peripheral $\mathrm{CD}^{+}$and $\mathrm{CD}^{+}$lymphocytes in SHIV-infected animals was determined by fluorescenceactivated cell sorter using fluorochrome-conjugated monoclonal antibodies as described previously. ${ }^{16}$

\section{Inoculation of Chinese Macaques With SHIV B'WHU $^{\prime}$}

Four juvenile Chinese rhesus macaques were infected

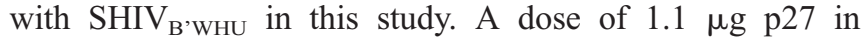
a volume of $15 \mathrm{~mL}$ of cell-free viral supernatant was infused intravenously via the femoral vein into a Chinese macaque (P1-\#0002). For serial passage, $10 \mathrm{~mL}$ of heparinized whole blood and $5 \mathrm{~mL}$ of bone marrow collected from the P1-\#0002 macaque at 2 weeks postinoculation were inoculated intravenously, also via the femoral vein, into another healthy Chinese macaque (P2-\#0296). Subsequent passages for P3 (P3-\#1032) and P4 (P4-\#1050) animals were carried out in the same manner as for P2-\#0296 animal. Our experimental protocols were reviewed and approved by the Institutional Animal Care and Use Committee at the Laboratory Animal Center in Wuhan University Laboratory Animal Center, where the experimental animals were housed.

\section{Viral Load Measurement}

The level of plasma viremia and viral RNA and proviral DNA loads in tissues was measured by a quantitative real-time PCR method as described previously. ${ }^{16,17}$ A molecular beaconbased real-time PCR assay was used to quantify the copy

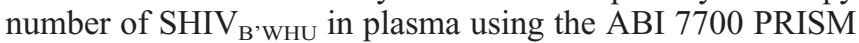
spectrofluorometric thermal cycler (Applied Biosystems, Foster City, CA). The limit of detection for plasma viral load was approximately 400 RNA copies per mililiter after the adjustment of the dilution factors.

\section{Immunohistochemistry}

Tissue sections from formalin-fixed paraffin-embedded small intestines (SIs) (jejunum) were stained with a mouse anti-human CD4 monoclonal antibody (NCL-CD4-1F6; Novacastra Laboratories, Ltd, London, United Kingdom) using the DakoCytomation-Labelled Streptavidin-Biotin2 System, Horseradish Peroxidase (LSAB2 System, HRP, DAKO Corporation, Carpinteria, CA) and AEC SubstrateChromogen solution (DAKO Corporation) as previously described. $^{21,22}$ 


\section{Determination of Coreceptor Usage}

The coreceptor usage of the postpassage SHIV $_{\mathrm{B}}$ 'WHU was determined by using GHOST CD4 cells expressing each of the following proteins including CCR1, CCR2b, CCR3, CCR4, CXCR4, CCR5, CCR8, Bob, Bonzo, and V28. Viral isolates were used to infect each of the cell types in duplicate in 96-well plates at a multiplicity of infection of 1.0. After incubation at $37^{\circ} \mathrm{C}$ for 2 hours, the cultures were washed 3 times with phosphate-buffered saline and maintained in the culture medium. The cells were photographed by fluorescent microscopy to detect GFP expression 24-48 hours postinoculation.

\section{RESULTS}

\section{Sequence Analysis of SHIV $\mathrm{B}^{\prime}$ WHuenv Gene}

To generate a subtype B' SHIV, we amplified the tat/rev/vpu/env fragments of HIV-1 subtype $\mathrm{B}$ ' ${ }_{\text {wHU }}$ from the proviral genome of a patient's PBMCs $\left(02 \mathrm{HN}_{\mathrm{SMX}}\right)$ by a nested PCR. Sequencing results indicated that the open-reading frames of tat/rev/vpu/env genes were complete without any frame shift. Because viral envelope determines viral genotype and phenotype, ${ }^{23}$ a phylogenetic analysis was conducted to determine the viral genotype as previously described. ${ }^{16}$ As

F1 depicted in the neighbor-joining tree (Fig. 1), the $\mathrm{SHIV}_{\mathrm{B} \text { 'WHU }}$ env clustered tightly with subtype B' strains identified either in Henan (02HNsmx2, 02HNsc11, and 02HNsq4), Yunnan (B'YN.RL42) of China or in other Southeast Asian countries such as Thailand (subtype B'.TH.93TH067) and Myanmar (subtype B'.MM.99.mSTD101). In contrast, env sequences from western countries, including those from the United States, are divergent from subtype B' virus (Fig. 1). ${ }^{2,24}$ Furthermore, the SimPlot and BootScan analysis showed that the env of SHIV $_{\mathrm{B} \text { 'wHU }}$ is a pure B' without cross over or recombination with viruses of other subtypes, including subtype C/B' viruses currently circulating in China. ${ }^{1,16}$ When compared with env genes of other SHIV strains including $\mathrm{SHIV}_{89.6}, \mathrm{SHIV}_{\mathrm{SF} 33}$, $\mathrm{SHIV}_{\mathrm{SF} 162}, \mathrm{SHIV}_{\mathrm{KU} 2}, \mathrm{SHIV}_{1157 \mathrm{ipd} 3 \mathrm{~N} 4}, \mathrm{SHIV}_{\mathrm{CHN} 19}, \mathrm{SHIV}_{\mathrm{MJ} 4}$, and $\mathrm{SHIV}_{\mathrm{E}-\mathrm{CAR}}$ (Fig. 1), ${ }^{18,25} \mathrm{SHIV}_{\mathrm{B} \text { 'WHU }}$ env is the only unique one representing the subtype $\mathrm{B}^{\prime}$ viral cluster. In addition, there were significant differences between SHIV $_{\mathrm{B} \text { 'wHU }}$ and other SHIVs especially in variable regions (V1, V2, V3, V4, and V5) based on the aligned amino acid sequences (See Figure, Supplemental Digital Content 1, http://links.lww.com/QAI/A28). The amino acid similarity between SHIV $_{\mathrm{B}}$ 'WHU, and each of these SHIV strains as mentioned above is $82 \%, 83 \%, 85 \%, 83 \%, 77 \%$, $77 \%, 73 \%$, and $75 \%$, respectively.

\section{Serial Passages of SHIV $_{B^{\prime} W H U}$ in Chinese Macaques}

We first determined that SHIV $_{\mathrm{B}^{\prime} \text { WHU }}$ used CCR5 as its coreceptor and was able to replicate in rhesus PBMCs (data not shown). To enhance the infectivity of $\mathrm{SHIV}_{\mathrm{B}}{ }^{\prime} \mathrm{wHU}$, in vivo serial passage of $\mathrm{SHIV}_{\mathrm{B}^{\prime} \text { 'WHU }}$ was performed in 4 naive Chinese rhesus macaques. ${ }^{16,26}$ Cell-free SHIV $_{\text {B'wHU supernatant was }}$ inoculated intravenously into a healthy Chinese rhesus monkey (P1-\#0002). Fourteen days after the inoculation, $10 \mathrm{~mL}$ of heparinized blood and $5 \mathrm{~mL}$ of bone marrow from this animal

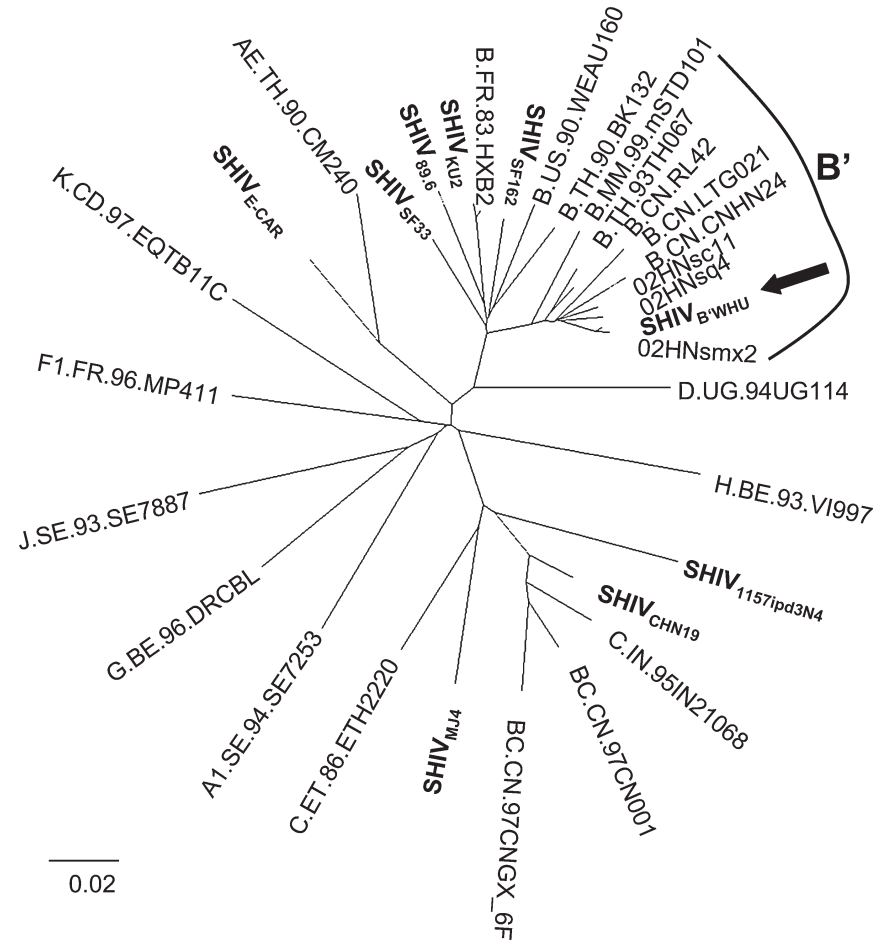

FIGURE 1. Phylogenetic analysis of the full-length env gene of $S_{\text {SHIV }}$ B'WHU. $^{\prime}$ SHIV $_{B^{\prime} W H U}$ is indicated by the arrow. The reference sequences were obtained from the HIV sequence database in GenBank. The tree was constructed by using full-length env nucleotide sequences by a neighbor-joining method as previously described. ${ }^{1,2}$ The length of branches is additive and represents genetic distance between pairs of viruses. The solid scale bar indicates a genetic distance of 0.02 . The reference SHIV strains are shown in bold.

was inoculated intravenously into a second naive animal (P2-\#0296). Such in vivo passage was carried out sequentially into the third and the fourth naive rhesus monkeys (P3-\#1032 and P4-\#1050), respectively. Unexpectedly, there was no detectable viremia in P1-\#0002 during the 14 days of infection (Fig. 2). P2 animal (P2-\#0296), however, developed productive infection as indicated by a burst of viremia $\left(10^{6}\right.$ copies of viral RNA per mL of plasma) 14 days after the blood-bone marrow transfusion. Further in vivo passages of $\operatorname{SHIV}_{\mathrm{B} \text { 'wHU have }}$ improved the viral infectivity because the burst of viral replication was detected earlier on day 12 with viral loads ranging $10^{6}$ to $10^{7}$ copies of viral RNA per milliliter of plasma (Fig. 2). During these 14 days of acute infection, there was no significant change in peripheral $\mathrm{CD}^{+}$and $\mathrm{CD}^{+} \mathrm{T}$-cell counts in all 4 infected Chinese rhesus macaques.

\section{Quantification of Proviral DNA and Viral RNA in Tissues}

Because SHIV $_{\mathrm{B} \text { 'wHU }}$ is a R5-tropic virus, we were curious to understand viral infection and pathology in the guts during the acute phase of infections, which has not been previously studied with any SHIVs before in vivo adaptation. Therefore, all animals were sacrificed 2 weeks postviral inoculation. Proviral DNA and viral RNA loads in tissues were 


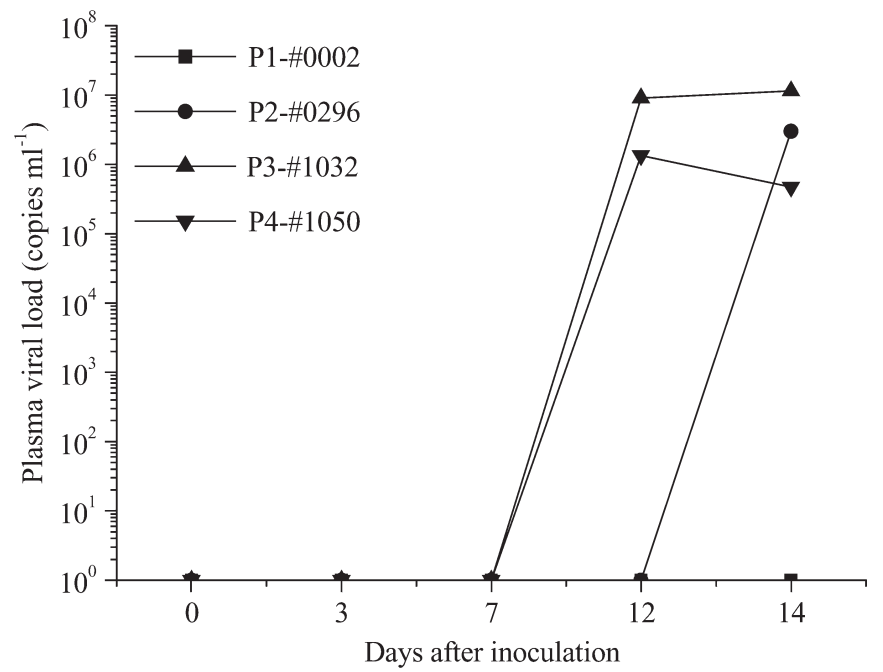

FIGURE 2. Characterization of plasma viral load in infected animals. The viral load of infected animals was measured using a real-time PCR assay. The $Y$ axis represents viral RNA copies per milliliter of plasma. The $X$ axis indicates the time postinoculation.

determined by a quantitative real-time PCR assay as previously described. ${ }^{17,27}$ In this analysis, SI, axillary lymph node (ALN) and mesenteric lymph node were examined in all animals (Fig. 3). Interestingly, despite the absence of detectable viral RNA in peripheral blood of P1-\#0002, there was significant level of viral RNA load and proviral DNA load in ALN of this animal (Fig. 3A). In P2-\#0296 monkey, accompanying the burst of viremia on day 14 post transfusion, proviral DNA and viral RNA were mainly detected in SIs in addition to low levels in ALN. In P3-\#1032 and P4-\#1050 monkeys, with the earlier burst of viremia on day 12 postinoculation, the viral RNA load and provial DNA load were significantly higher in SIs. Moreover, both proviral DNA and viral RNA load were significantly higher in mesenteric lymph nodes (Fig. 3B), which belongs to mucosal tissue compartments. It is possible that the enhanced viral infectivity is related to the increasing infection of $\mathrm{CD}^{+} \mathrm{T}$ lymphocytes in mucosal tissue compartments. As for the failure of detectable viral load in other tissues, we believed that it was probably due to the limit of our viral load assay because viral genes were readily detected in all tissue compartments tested by nested PCR.

\section{Immunohistochemistry Analysis of CD4 ${ }^{+}$ T Cells in Sls}

To further determine the pathogenicity of CCR5-tropic

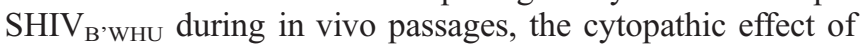
viral infection was investigated in the SIs. We were unable to analyze the $\mathrm{CD}^{+} \mathrm{T}$ cells using the flow cytometry technique due to the lack of equipment at our study site. Immunohistochemistry analysis, however, was performed on formalinfixed paraffin-embedded SIs to determine the loss of $\mathrm{CD}^{+} \mathrm{T}$ cells using a mouse anti-human CD4 monoclonal antibody. ${ }^{22}$ $\mathrm{CD}^{+} \mathrm{T}$ cells were stained red as shown in Fig. 4. For each monkey, we analyzed multiple tissue sections and multiple fields of each tissue section. Compared with uninfected rhesus monkeys, the number of $\mathrm{CD}^{+}{ }^{+} \mathrm{T}$ cells in $\mathrm{SHIV}_{\mathrm{B}}{ }^{\prime} \mathrm{WHU}$-infected monkeys was significantly reduced especially among the intraepithelial lymphocytes (IEL) of SIs. CD4 ${ }^{+} \mathrm{T}$ cells were detected among the lamina propria lymphocytes but with reduced numbers, and in mucosa-associated lymphocyte aggregates or tissues (Fig. 4).

\section{Unchanged CCR5-Tropism of SHIV ${ }_{B^{\prime} W H U}$ After Serial Passage}

To determine the viral coreceptor usage, viruses were reisolated from $\mathrm{P} 2-\mathrm{P} 4$ passaged monkeys. These viral isolates were used to infect GHOST CD4 cells expressing each of the coreceptors, including CCR1, CCR2b, CCR3, CCR4, CXCR4, CCR5, CCR8, Bob, Bonzo, and V28. These isolates
FIGURE 3. The quantification of viral RNA (top) and proviral DNA (bottom) in different tissues: Axillary LN $(A, D)$, Mesenteric LN (B, E), and SI (C, F). Viral RNA copies (GAPDH copies $)^{-1}$ and proviral DNA copies $(\mu \mathrm{g} \mathrm{DNA})^{-1}$ were determined by real-time PCR assays. The error bar indicates the experimental variation, whereas the dot line indicates the cut-off value. Viral env genes were amplified by nested PCR for sequence analysis from all tissue compartments as shown here. NC refers to uninfected negative control.
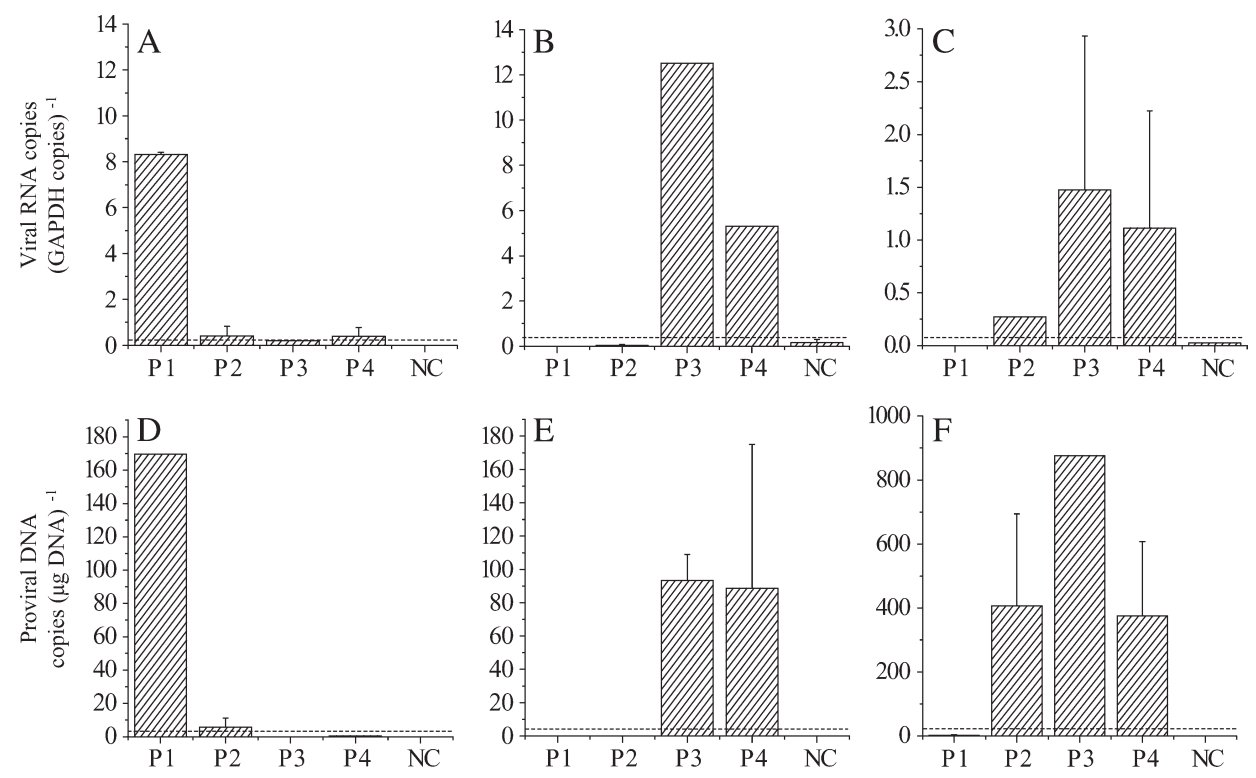

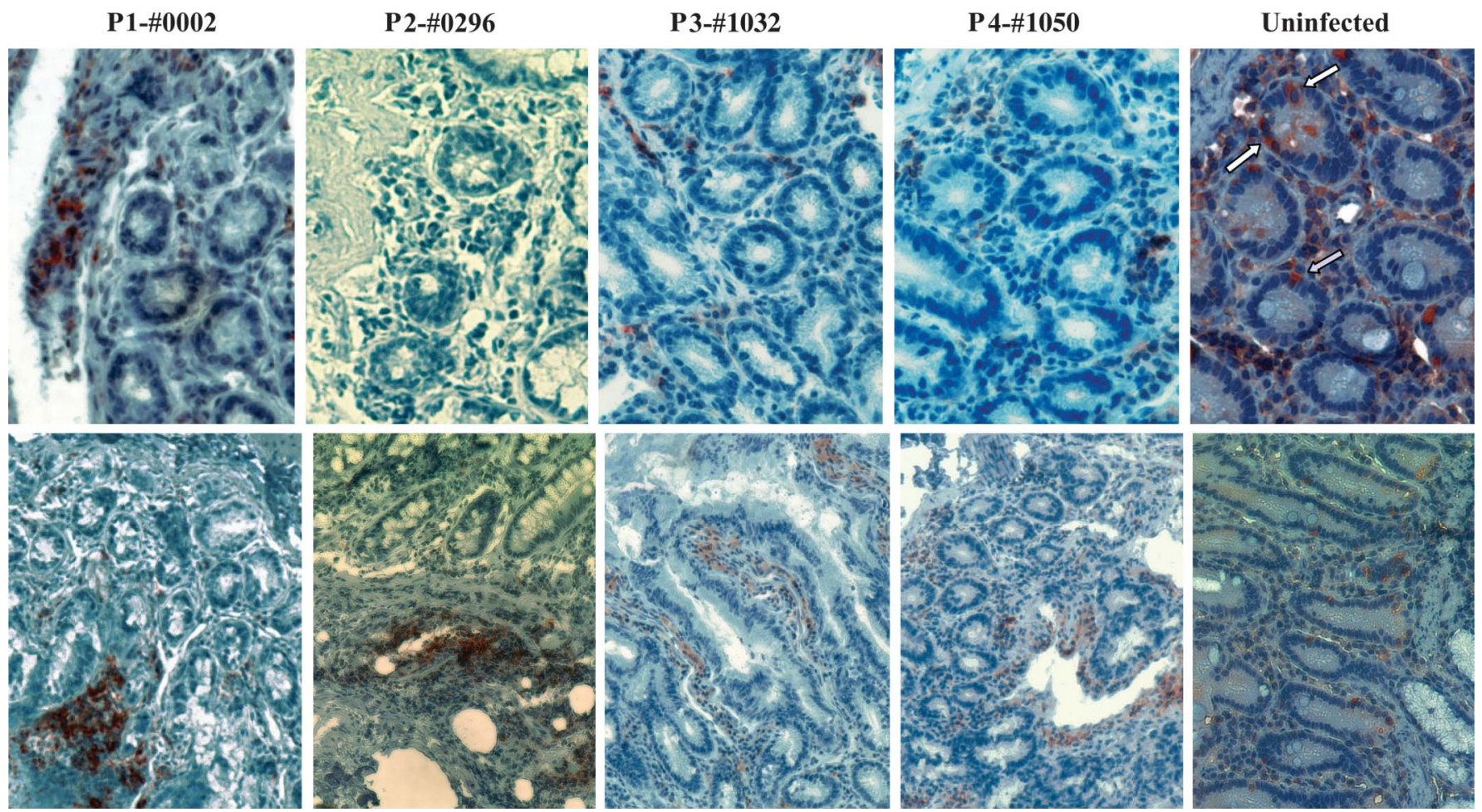

FIGURE 4. Immunohistochemistry analysis of CD4 ${ }^{+}$IEL loss in SIs of infected macaques. CD4 ${ }^{+}$T lymphocytes were detected in SIs of infected and control macaques with a mouse anti-human CD4 monoclonal antibody at 2 weeks postinfection. CD4 ${ }^{+}$cells are in red. The arrows showed the CD4+ IELs. At least 5 tissue sections and multiple fields of each section were analyzed for each monkey. Original magnifications: $\times 400$ (top panel) and $\times 200$ (bottom panel).

infected GHOST CD4 Hu-CCR5 cells but not other cells (See Figure, Supplemental Digital Content 2, http://links.lww.com/QAI/A29). with a pattern similar to that of the parental $\mathrm{SHIV}_{\mathrm{B}}{ }^{\prime}$ wHu. Furthermore, there were only low frequent sporadic amino acid substitutions identified in their env fragments. These results suggest that, despite in vivo serial passage, $\mathrm{SHIV}_{\mathrm{B}^{\prime} \text { WHU }}$ has maintained CCR5 specificity with no evidence of altered coreceptor utilization.

\section{DISCUSSION}

Over the past years, Asia has become the second most heavily affected continent by HIV/AIDS in the world. Because Asia has the world's largest human population, effective measures must be implemented to control the increasing prevalence of HIV-1 infection. To this end, the development of a relevant animal model to facilitate the testing of various preventive measures (eg vaccine, microbicide, antivirals) remains necessary. HIV-1 subtype B' is a major HIV-1 genotype not only in China but also in other Southeast Asia countries. ${ }^{2,28}$ Because there is a significant amount of sequence variation in env genes of subtype B' and western B viruses (See Figure, Supplemental Digital Content 1, http://links.lww.com/QAI/A28). currently available B SHIVs are not necessarily suitable for studies of subtype B' viral transmission and pathogenesis. For example, recent studies indicated that some subtype B' HIV-1 strains are less susceptible to a few potent neutralizing antibodies derived from patients infected with western B viruses. ${ }^{29}$ The newly constructed
SHIV $_{\mathrm{B} \text { 'WHU }}$ is therefore particularly useful for studying issues related to subtype B' $e n v$-mediated infection in a nonhuman primate model.

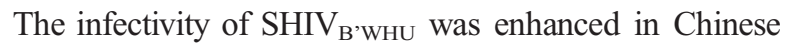
macaques after in vivo serial passage. Before in vivo passage, the phenotype and replication kinetics of $\mathrm{SHIV}_{\mathrm{B} \text { 'wHU was }}$ determined by in vitro assays. SHIV $\mathrm{B}_{\mathrm{B} \text { whU }}$ was found to be CCR5-tropic similar to its primary parental HIV-1 isolate (See Figure A, Supplemental Digital Content 3, http://links.lww.com/QAI/A30). This phenotype is crucial because it represents the dominant type of sexually transmitted HIV-1 strains. ${ }^{23,30}$ Furthermore, SHIV $_{\text {B'wHU was demonstrated }}$ to be replication-competent in PBMCs of 2 randomly selected Chinese macaques (See Figure B, Supplemental Digital Content 3, http://links.lww.com/QAI/A30), which warranted the subsequent in vivo experiments. Although no detectable viremia was found in peripheral blood of P1-\#0002 monkey at the acute phase of infection, the subsequent increasing viremia detected in P2-P4 animals suggested that $\mathrm{SHIV}_{\mathrm{B}}{ }^{\prime}$ wHU has adapted to grow in vivo and therefore became infectious in Chinese macaques (Fig. 2). In general, the observed pattern of $\mathrm{SHIV}_{\mathrm{B}}{ }^{\prime} \mathrm{WHU}$ replication in macaques is similar to previous findings of SHIV infection in a new monkey host. ${ }^{16}$ Consistently, the improved growth kinetics and the broader tissue tropism of

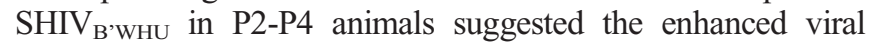
infectivity in vivo (Figs. 2, 3). The enhanced infectivity, however, did not result in altered CCR5-tropism and therefore, unlike pathogenic X4-tropic and X4R5-tropic SHIV strains which 
induce severe $\mathrm{CD}^{+}$T-cell loss in the periphery, ${ }^{23,31}$ no significant $\mathrm{CD}^{+}$lymphocytopenia was found in peripheral blood of $\mathrm{SHIV}_{\mathrm{B}}{ }^{\prime} \mathrm{wHU}^{-i n f e c t e d ~ m a c a q u e s . ~ I n ~ a d d i t i o n, ~ a l t h o u g h ~}$ we see enhanced viral replications in P3 and P4 macaques, we cannot exclude the possibility that the target cells of P1 and P2 are less susceptible to SHIV $_{\mathrm{B}^{\prime} \text { WHU }}$ infection.

The rapid in vivo serial passage improved the infectivity of SHIV $_{\text {B'WHU }}$ probably by improving viral fitness and extending the involvement of tissue $\mathrm{CD}^{+} \mathrm{T}$ lymphocytes in vivo. Previous investigators demonstrated that serial passage of SHIV in macaques resulted in virus with high pathogenecity. ${ }^{18,32,33}$ The pathogenicity was often indicated by high viral load, $\mathrm{CD} 4^{+} \mathrm{T}$ lymphocytopenia, and even simian AIDS. In this study, we found that a freshly made (before adaptation in macaques) CCR5-tropic SHIV was able to deplete $\mathrm{CD}^{+}$IELs and to less extent the $\mathrm{CD}^{+}$lamina propria lymphocytes in all 4 macaques tested. This finding suggested that $\mathrm{CD} 4^{+}$IELs were probably more susceptible to the acute infection of SHIV $_{B^{\prime} \text { wHU }}$ even without the overtime adaptation of the virus in vivo. However, the number of experimental animals is small in our study. Because similar studies were not conducted for other CCR5-tropic SHIVs, it remains to be determined whether our finding is a general phenomenon for viruses of the same phenotype. Moreover, a more quantitative FACS analysis is needed to determine the quantitative loss of $\mathrm{CD}^{+}$IEL in SHIV $_{\mathrm{B}^{\prime} \text { WHU-infected animals in future study. Previous studies }}$ indicated that some changes were also detected in the IELs population during SIV infection, ${ }^{34}$ it might be critical to determine the role of IELs in seeding the early transmission and replication of CCR5-tropic HIV-1. Because the viral tropism was not changed in P3 and P4 monkeys, it is therefore possible that the improved infectivity of $\mathrm{SHIV}_{\mathrm{B}}$ 'WHU was not simply due to its phenotypic changes. It is likely that the early involvement of $\mathrm{CD}^{+} \mathrm{T}$ lymphocytes in broad tissue compartments also contributed to the rapid burst of viremia especially in P3 and P4 animals (Fig. 3). To confirm the improved infectivity of the virus, 3 additional Chinese macaques were recently inoculated with SHIV ${ }_{\text {B'WHU }}$ derived from the P4 animal. All 3 animals have become infected as indicated by peak viremia and subsequent seroconversion. These animals will be used to determine whether or not $\mathrm{SHIV}_{\mathrm{B} \text { 'WHU }}$ would lead to persistent infection and subsequent simian AIDS in Chinese macaques. In addition, to build a useful $\mathrm{SHIV}_{\mathrm{B}^{\prime} \mathrm{WHU}} /$ Chinese macaque model, it would also be necessary to determine the mucosal transmissibility of $\mathrm{SHIV}_{\mathrm{B} \text { 'WHU }}$ with enhanced infectivity in future studies. Lastly, because different macaque species may respond to SIV or SHIV infection differently, future studies should determine the pathogenicity and disease course of $\mathrm{SHIV}_{\mathrm{B}}$ wHU infection in Indian or pig-tailed macaques.

\section{ACKNOWLEDGMENTS}

We thank Drs D. D. Ho and K. Y. Yuen for scientific advice and C. Cheng-Mayer for $\mathrm{SHIV}_{33}$ molecular clone and discussions.

\section{REFERENCES}

1. Zhang L, Chen Z, Cao Y, et al. Molecular characterization of human immunodeficiency virus type 1 and hepatitis $\mathrm{C}$ virus in paid blood donors and injection drug users in china. J Virol. 2004;78:13591-13599.
2. Su B, Liu L, Wang F, et al. HIV-1 subtype B' dictates the AIDS epidemic among paid blood donors in the Henan and Hubei provinces of China. AIDS. 2003;17:2515-2520.

3. Volkow P, Del Rio C. Paid donation and plasma trade: unrecognized forces that drive the AIDS epidemic in developing countries. Int J STD AIDS. 2005;16:5-8.

4. Zhang LF, Qiao XC, Nie XY, et al. A retrospective cohort study on the survival of blood-borne human immunodeficiency virus cases in a county, China [in Chinese]. Zhonghua Liu Xing Bing Xue Za Zhi. 2004;25:941-944.

5. Zhuang K, Gui XE, Luo JL, et al. A survey of children with HIV/AIDS in highly epidemic villages of AIDS [in Chinese]. Zhonghua Er Ke Za Zhi. 2003;41:586-589.

6. Zhuang K, Gui X, Su B, et al. High prevalence of HIV infection among women and their children in Henan Province, China. J Acquir Immune Defic Syndr. 2003;33:649-650

7. Cohen J. HIV/AIDS in China. An unsafe practice turned blood donors into victims. Science. 2004;304:1438-1439.

8. Beach MV. "Blood heads" and AIDS haunt China's countryside. Lancet. 2001;357:49.

9. Huang Y, Henderson GE, Pan S, et al. HIV/AIDS risk among brothelbased female sex workers in China: assessing the terms, content, and knowledge of sex work. Sex Transm Dis. 2004;31:695-700.

10. Chen WT, Han M, Holzemer WL. Nurses' knowledge, attitudes, and practice related to HIV transmission in northeastern China. AIDS Patient Care STDS. 2004;18:417-422.

11. Motohara M, Ibuki K, Miyake A, et al. Impaired T-cell differentiation in the thymus at the early stages of acute pathogenic chimeric simian-human immunodeficiency virus (SHIV) infection in contrast to less pathogenic SHIV infection. Microbes Infect. 2006;8:1539-1549.

12. Akiyama H, Ishimatsu M, Miura T, et al. Construction and infection of a new simian/human immunodeficiency chimeric virus (SHIV) containing the integrase gene of the human immunodeficiency virus type 1 genome and analysis of its adaptation to monkey cells. Microbes Infect. 2008;10: 531-539.

13. Bona R, Macchia I, Baroncelli S, et al. T cell receptor excision circles (TRECs) analysis during acute intrarectal infection of cynomolgus monkeys with pathogenic chimeric simian human immunodeficiency virus. Virus Res. 2007;126:86-95.

14. Luciw PA, Pratt-Lowe E, Shaw KE, et al. Persistent infection of rhesus macaques with T-cell-line-tropic and macrophage-tropic clones of simian/ human immunodeficiency viruses (SHIV). Proc Natl Acad Sci U S A. 1995;92:7490-7494.

15. Shibata R, Siemon C, Czajak SC, et al. Live, attenuated simian immunodeficiency virus vaccines elicit potent resistance against a challenge with a human immunodeficiency virus type 1 chimeric virus. J Virol. 1997;71:8141-8148.

16. Chen Z, Huang Y, Zhao X, et al. Enhanced infectivity of an R5-tropic simian/human immunodeficiency virus carrying human immunodeficiency virus type 1 subtype $\mathrm{C}$ envelope after serial passages in pig-tailed macaques (Macaca nemestrina). J Virol. 2000;74:6501-6510.

17. Chen Z, Zhao X, Huang Y, et al. CD4+ lymphocytopenia in acute infection of Asian macaques by a vaginally transmissible subtype-C, CCR5-tropic Simian/Human Immunodeficiency Virus (SHIV). J Acquir Immune Defic Syndr. 2002;30:133-145.

18. Himathongkham S, Halpin NS, Li J, et al. Simian-human immunodeficiency virus containing a human immunodeficiency virus type 1 subtype$\mathrm{E}$ envelope gene: persistent infection, CD4(+) T-cell depletion, and mucosal membrane transmission in macaques. $J$ Virol. 2000;74: 7851-7860

19. Page RD. TreeView: an application to display phylogenetic trees on personal computers. Comput Appl Biosci. 1996;12:357-358.

20. Lole KS, Bollinger RC, Paranjape RS, et al. Full-length human immunodeficiency virus type 1 genomes from subtype $\mathrm{C}$-infected seroconverters in India, with evidence of intersubtype recombination. J Virol. 1999;73:152-160.

21. Igarashi T, Brown CR, Byrum RA, et al. Rapid and irreversible CD4+ T-cell depletion induced by the highly pathogenic simian/human immunodeficiency virus SHIV(DH12R) is systemic and synchronous. J Virol. 2002;76:379-391.

22. Fukazawa Y, Miyake A, Ibuki K, et al. Small intestine CD4+ T cells are profoundly depleted during acute simian-human immunodeficiency virus infection, regardless of viral pathogenicity. J Virol. 2008;82:6039-6044. 
23. Harouse JM, Gettie A, Tan RC, et al. Distinct pathogenic sequela in rhesus macaques infected with CCR5 or CXCR4 utilizing SHIVs. Science. 1999; 284:816-819.

24. Graf M, Shao Y, Zhao Q, et al. Cloning and characterization of a virtually full-length HIV type 1 genome from a subtype B'-Thai strain representing the most prevalent B-clade isolate in China. AIDS Res Hum Retroviruses. 1998; 14:285-288.

25. Song RJ, Chenine AL, Rasmussen RA, et al. Molecularly cloned SHIV1157ipd3N4: a highly replication-competent, mucosally transmissible R5 simian-human immunodeficiency virus encoding HIV clade C Env. J Virol. 2006;80:8729-8738.

26. Borsetti A, Baroncelli S, Maggiorella MT, et al. Viral outcome of simianhuman immunodeficiency virus SHIV-89.6P adapted to cynomolgus monkeys. Arch Virol. 2008;153:463-472.

27. Miyake A, Ibuki K, Enose Y, et al. Rapid dissemination of a pathogenic simian/human immunodeficiency virus to systemic organs and active replication in lymphoid tissues following intrarectal infection. J Gen Virol. 2006;87:1311-1320.

28. Deng X, Liu H, Shao Y, et al. The epidemic origin and molecular properties of B': a founder strain of the HIV-1 transmission in Asia. AIDS. 2008;22:1851-1858.
29. Chong H, Hong $\mathrm{K}$, Zhang $\mathrm{C}$, et al. Genetic and neutralization properties of HIV-1 env clones from subtype B/BC/AE infections in China. J Acquir Immune Defic Syndr. 2008;47:535-543.

30. Nishimura Y, Igarashi T, Donau OK, et al. Highly pathogenic SHIVs and SIVs target different CD4+ T cell subsets in rhesus monkeys, explaining their divergent clinical courses. Proc Natl Acad Sci U S A. 2004;101: 12324-12329.

31. Klinger JM, Himathongkham S, Legg H, et al. Infection of baboons with a simian immunodeficiency virus/HIV-1 chimeric virus constructed with an HIV-1 Thai subtype E envelope. AIDS. 1998;12: 849-857.

32. Joag SV, Li Z, Foresman L, et al. Chimeric simian/human immunodeficiency virus that causes progressive loss of CD4+ T cells and AIDS in pig-tailed macaques. $J$ Virol. 1996;70:3189-3197.

33. Reimann KA, Li JT, Veazey R, et al. A chimeric simian/human immunodeficiency virus expressing a primary patient human immunodeficiency virus type 1 isolate env causes an AIDS-like disease after in vivo passage in rhesus monkeys. $J$ Virol. 1996;70:6922-6928.

34. Veazey RS, DeMaria M, Chalifoux LV, et al. Gastrointestinal tract as a major site of CD4+ $\mathrm{T}$ cell depletion and viral replication in SIV infection. Science. 1998;280:427-431. 